\title{
Estrogen metabolism genotypes, use of long-term hormone replacement therapy and risk of postmenopausal breast cancer
}

\author{
JASMINA ZIVA CERNE ${ }^{1}$, SRDJAN NOVAKOVIC ${ }^{2}$, SNJEZANA FRKOVIC-GRAZIO $^{3}$, \\ MAJA POHAR-PERME ${ }^{4}$, VIDA STEGEL ${ }^{2}$ and KSENIJA GERSAK ${ }^{1}$ \\ ${ }^{1}$ Institute of Medical Genetics, Department of Obstetrics and Gynecology, University Medical Centre Ljubljana, \\ 1000 Ljubljana; Departments of ${ }^{2}$ Molecular Diagnostics and ${ }^{3}$ Pathology, Institute of Oncology, \\ 1000 Ljubljana; ${ }^{4}$ Institute for Biostatistics and Medical Informatics, Faculty of Medicine, \\ University of Ljubljana, 1000 Ljubljana, Slovenia
}

Received February 9, 2011; Accepted March 24, 2011

DOI: $10.3892 /$ or.2011.1298

\begin{abstract}
Association between long-term hormone replacement therapy (HRT) use and increased risk of breast cancer is still under debate. Functionally relevant genetic variants within the estrogen metabolic pathway may alter exposure to exogenous sex hormones and affect the risk of postmenopausal breast cancer. We investigated the associations of common polymorphisms in 4 genes encoding key proteins of the estrogen metabolic pathway, duration of HRT use and their interactions with breast cancer risk. We studied 530 breast cancer cases and 270 controls of the same age and ethnicity participating in a case-control study of postmenopausal women. Duration of HRT use was ascertained through a postal questionnaire. Genotyping was conducted for CYP1B1 (rs1056836), COMT (rs4680), GSTP1 (rs1695) and MnSOD (rs4880) polymorphisms by PCR-based RFLP and TaqMan ${ }^{\circledR}$ allelic discrimination method. Adjusted odds ratios and $95 \%$ confidence intervals were calculated using logistic regression analysis. HRT use was significantly associated with decreased breast cancer risk $(\mathrm{p}<0.001)$. None of the polymorphisms studied was associated with breast cancer risk. A significant interaction was observed between MnSOD 47T $>C$ and HRT use $\left(p_{\text {interaction }}=0.036\right)$; the risk of breast cancer associated with long-term vs. short-term HRT use was decreased in women homozygous for the wild-type allele and increased in women with at least one variant allele of the MnSOD 47T $>$ C polymorphism. Our results suggest that MnSOD 47T $>$ C polymorphism in interaction with long-term HRT use may modify the risk of breast cancer.
\end{abstract}

Correspondence to: Dr Ksenija Gersak, Institute of Medical Genetics, Department of Obstetrics and Gynecology, University Medical Centre Ljubljana, Slajmerjeva 3, 1000 Ljubljana, Slovenia E-mail: ksenija.gersak@mf.uni-lj.si

Key words: breast cancer, estrogen metabolism, polymorphisms, hormone replacement therapy use, association study

\section{Introduction}

Exposure to estrogens has been associated with an increased risk of developing breast cancer (1). The mechanisms through which estrogens contribute to the carcinogenic process are complex. Evidence suggests the involvement of estrogenreceptor-mediated genomic and nongenomic signaling which increases cell proliferation of mammary tissue. Furthermore, the metabolism of estrogens leads to the production of catechol estrogens (CE), and the more reactive semiquinone and quinone metabolites, which exert genotoxic effects (2). To date, no studies have definitively demonstrated that estrogen metabolites contribute to breast cancer incidence, although two lines of evidence support this possibility (1). First, estrogen metabolites and conjugates have been detected in human breast tissue (3). Higher levels of activating 4-hydroxyestrogens [4-OH-E1(E2)] and lower levels of protecting methoxy-catechol estrogens $\left[\mathrm{CH}_{3} \mathrm{O}-\mathrm{E} 1(\mathrm{E} 2)\right]$ were detected in breast cancer tissue. In contrast, in breast tissue of women without breast cancer, higher levels of protecting 2-OH$\mathrm{E} 1(\mathrm{E} 2)$ and $\mathrm{CH}_{3} \mathrm{O}-\mathrm{E} 1(\mathrm{E} 2)$ were found. The level of CE-quinone conjugates in breast cancer patients was three times that in the controls, suggesting there was a higher probability in the cases for quinones to react with DNA and generate mutations that may initiate cancer (Fig. 1).

The second line of evidence supporting the involvement of estrogen metabolites in the development of human breast cancer comes from association studies on breast cancer risk and polymorphisms in candidate polymorphic genes (5). These include genes encoding for enzymes involved in the aforementioned estrogen metabolism and in the detoxification of reactive oxygen species (ROS) emerging in these reactions.

Several observational epidemiologic studies (6-9) and a randomized clinical trial (10) have investigated the effects of postmenopausal HRT on various health outcomes, including breast cancer. They all suggest that HRT is effective for the short-term relief of menopausal symptoms. However, the longterm use of HRT ( $>5$ years) is associated with an elevated breast cancer risk with combined estrogen plus progestin 


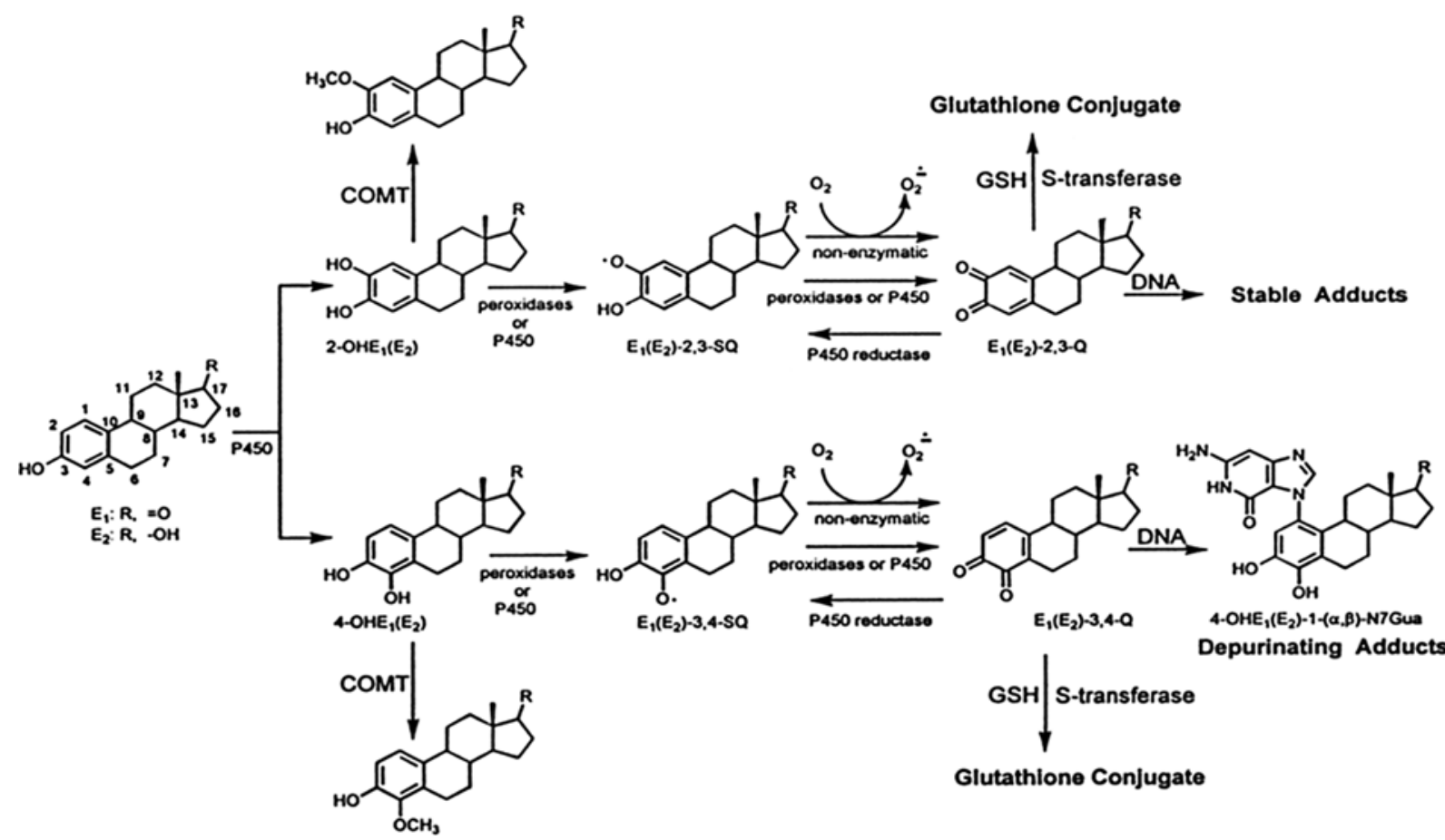

Figure 1. Activating and deactivating (protective) pathways of estrogen metabolism and formation of DNA adducts, modified from Cavalieri et al. (4).

therapy posing a greater risk than estrogen monotherapy. This higher risk is noted with various types of preparations and different routes of administration.

Modification of the effect of HRT use on breast cancer risk by polymorphisms in genes involved in metabolism of estrogens is thus highly reasonable, although currently limited. Further insight into this is important, as it may eventually result in the ability to identify postmenopausal women who are particularly susceptible to breast cancer when exposed to surplus exogenous hormones for longer periods. Therefore, the purpose of this study was to evaluate the associations of common polymorphisms in 4 estrogen metabolic pathway genes, duration of HRT use, as well as their interactions with breast cancer risk in a case-control study of postmenopausal women. Each of the selected genes, CYP1B1, COMT, GSTP1 and MnSOD, is highly expressed in breast tissue and involved in a distinct estrogen metabolic sub-pathway.

\section{Patients and methods}

Study population. A breast cancer case-control study was conducted in Slovenia between January 1, 2006 and December 31, 2008. Eligible cases were postmenopausal women diagnosed with invasive primary breast cancer at the Institute of Oncology in Ljubljana, Slovenia, who were 50-69 years of age at the time of diagnosis and of Caucasian ethnic origin. Eligible controls were postmenopausal women randomly selected from the outpatient clinic records of the Department of Obstetrics and Gynecology, University Medical Centre Ljubljana, Slovenia, who were 50-69 years of age, of Caucasian ethnic origin and without a history of breast cancer. Informed written consent was obtained from all of the women enrolled in the study.
Questionnaire. Both cases and controls were invited to participate in the study via a personal letter and asked to complete the enclosed written questionnaire. In addition to general information (socioeconomic status, weight, height), the questionnaire contained questions requesting reproductive data (age at menarche, number of pregnancies, age at first delivery, number of deliveries, breastfeeding, age at menopause), family history of breast and ovarian cancer (first-degree relatives), smoking and alcohol consumption. Detailed questions were asked regarding drug intake, sex hormones in particular (oral contraceptive (OC) and HRT use). A color chart displaying all preparations ever marketed in Slovenia was included in the questionnaire to aid recall. Information was obtained regarding years of OC use ( 4 categories: $<1,1$ to $<5,5$ to $<10$, $\geq 10$ ), years of HRT use ( 3 categories: no use, $<1$; short-term use, 1 to $<5$; long-term use, $\geq 5$ ) and regimen of HRT use (estrogen therapy, estrogen plus progestin therapy). OC and HRT use for $<1$ year was considered no use. Women were assumed to be postmenopausal if they reported their last natural menstrual bleeding at least 12 months before the reference date or had undergone a bilateral oophorectomy. The study protocol was approved by the National Medical Ethics Committee of the Republic of Slovenia (no. 61/06/07).

Specimen collection and isolation of DNA. In the patients, DNA was extracted from formalin-fixed paraffin-embedded normal breast tissues using the HP PCR Template Preparation Kit (Roche Diagnostics GmbH, Mannheim, Germany) following the manufacturer's protocol. The control group women were invited to provide blood sample, and genomic DNA was extracted from peripheral blood lymphocytes using FlexiGene DNA Kit 250 (Qiagen GmbH, Hilden, Germany) following the manufacturer's protocol. 
Genotyping. Genotyping for the polymorphism c. $1294 \mathrm{C}>\mathrm{G}$ (p. Leu432Val) in gene CYP1B1 was carried out using the PCR-based RFLP method. Each PCR product was digested with restriction endonuclease Eco57I (Fermentas International Inc., Burlington, Canada), and DNA fragments were separated and visualized by electrophoresis on polyacrylamide gels. Genotyping for polymorphisms c. 472G>A (p. Val108/158Met) in gene COMT, c. 313A $>\mathrm{G}$ (p. Ile105Val) in gene GSTP1 and c. $47 \mathrm{~T}>\mathrm{C}$ (p. Val16Ala) in gene MnSOD was performed on 96-well plates using fluorogenic 5'-nuclease assays on a LightCycler ${ }^{\circledR} 480$ System (Roche Diagnostics GmbH). Each reaction mix contained genomic DNA, LightCycler 480 Probes Master (Roche Diagnostics GmbH) and Custom TaqMan SNP Genotyping Assay (Applied Biosystems, Werterstadt, Germany). All genotyping protocols (PCR reaction conditions, primers and probes) can be provided upon request from the corresponding author. Positive control samples (homozygote for wild-type allele, heterozygote, homozygote for variant allele) and negative control sample were included in each batch of samples. Gels were scored by two different readers, and discordant samples were repeated. Apart from CYP1B1 1294C $>$ G, all polymorphisms had no samples that failed to be genotyped. For CYP1B1 $1294 \mathrm{C}>\mathrm{G}, 1 \%$ of the samples failed. Samples that failed to be genotyped were scored as missing. Reliability was assessed by random selection of $5 \%$ of samples in which all genotypes were confirmed by sequencing using ABI PRISM 7000 sequence detection system (Applied Biosystems). Concordance was $100 \%$ for all genotypes.

Statistical analyses. T-tests (for means) and Chi-square tests (for frequencies) were carried out to detect differences in baseline characteristics between cases and controls. Observed genotype frequencies were tested for deviation from HardyWeinberg equilibrium with the Chi-square goodness-of-fit test. Odds ratios (ORs) for breast cancer risk and the corresponding $95 \%$ confidence intervals (CI) were calculated using logistic regression analysis. The homozygous wild-type genotype, as determined by the more common of the homozygous genotypes, served as a reference category, with the heterozygous genotype and homozygous variant genotypes being combined into 1 category. Effect modification by the different genetic variants was investigated for the association between HRT use (no use, 0 to $<1$; short-term use, 1 to $<5$; long-term use, $\geq 5$ ) and breast cancer risk. Variables which significantly altered the magnitude of the risk were included in the final model. Analyses conducted to evaluate associations between the different genetic variants and breast cancer risk were adjusted for confounding effects of body mass index (BMI) $\left(<25,25\right.$ to $\left.<30, \geq 30 \mathrm{~kg} / \mathrm{m}^{2}\right)$, years of OC use (never or $<1,1$ to $<5,5$ to $<10, \geq 10$ years), years of HRT use (never or $<1,1$ to $<5, \geq 5$ years) and smoking at the time of diagnosis (non-smokers, 1 to $<10$ cigarettes per day, $\geq 10$ cigarettes per day). All other analyses were adjusted for confounding effects of BMI $\left(<25,25\right.$ to $\left.<30, \geq 30 \mathrm{~kg} / \mathrm{m}^{2}\right)$, years of OC use (never or $<1,1$ to $<5,5$ to $<10, \geq 10$ years) and smoking at the time of diagnosis (non-smokers, 1 to $<10$ cigarettes per day, $\geq 10$ cigarettes per day). We also considered age, education level and all other known reproductive breast cancer risk factors (age at menarche, age at first full-term pregnancy, parity, breastfeeding and age at menopause) as potential confounding factors, but none of these covariates had substantial effect on the ORs. Thus, we report the results without adjustments for these factors. All statistical analyses were carried out using the SPSS 18.0 software package. $\mathrm{P}<0.05$ was considered statistically significant.

\section{Results}

The response rates were $82.5 \%(825 / 1000)$ for cases and $73.2 \%(732 / 1000)$ for controls. Complete data for all variables considered in the multivariate model were available for 784 cases and 709 controls. Blood samples were provided only by $38.1 \%(270 / 709)$ of the control group women. The number of cases included in the genotype analyses was therefore proportionally decreased by random selection to gain a $2: 1$ ratio in case-control comparisons. The final analysis thus included 800 postmenopausal women aged 50-69 years: 530 were diagnosed with primary breast cancer and 270 were healthy volunteers (control group). The mean age for cases was $60.45 \pm 5.84$ years and for controls $60.1 \pm 5.85$ years, and did not differ significantly between the groups $(\mathrm{p}=0.432)$.

Distribution of the selected characteristics for cases and controls was consistent with most established risk factors and is documented in Table I. Rather unexpectedly, significantly more women in the control group were using HRT $(65.8 \%$ of controls vs. $29.6 \%$ of cases). Although the difference did not reach statistical significance, combined HRT was prescribed to a higher percentage of cases, whereas estrogen only HRT to a higher percentage of controls.

The genotype frequencies among cases and controls were consistent with those predicted by Hardy-Weinberg equilibrium apart for GSTP1 $(\mathrm{p}=0.04)$. When adjusted for confounding effects, none of the 4 studied genetic variants (CYP1B1 1294C>G, COMT 472G >A, GSTP1 313A>G, MnSOD 47T $>$ C) was associated with postmenopausal breast cancer risk. All of the variant alleles appeared to pose a slightly increased risk for breast cancer, but the effects were not statistically significant (data not shown). On the other hand, HRT use was significantly associated with decreased breast cancer risk (Table II).

Additionally, we investigated whether the functionally relevant polymorphisms within the estrogen metabolic pathway modify the exposure to exogenous sex hormones and thus affect the risk of postmenopausal breast cancer. A significant interaction between MnSOD 47T $>\mathrm{C}$ and duration of HRT use was observed $\left(p_{\text {interaction }}=0.036\right)$; the risk of breast cancer associated with long-term vs. short-term HRT use decreased in women homozygous for the wild-type allele and increased among women with at least one variant allele of the MnSOD 47T $>$ C polymorphism (Table III). No significant interaction was found between different genetic variants and breast cancer risk with respect to regimen of HRT (estrogen monotherapy vs. combined, estrogen plus progestin therapy) (data not shown).

\section{Discussion}

In this case-control study of postmenopausal Caucasian women, we investigated associations of functionally relevant genetic variants in 4 genes encoding key proteins of the estrogen metabolic pathway, duration of HRT use, as well as their 
Table I. Characteristics of the study population.

\begin{tabular}{|c|c|c|c|}
\hline Variable & Cases $(n=530)$ & Controls $(n=270)$ & p-value \\
\hline \multicolumn{4}{|l|}{ Education, highest degree obtained (\%) } \\
\hline Primary school & 30.7 & 6.3 & \multirow[t]{3}{*}{$<0.001$} \\
\hline Secondary school & 59.2 & 70.3 & \\
\hline University, $\mathrm{PhD}$ & 10.1 & 23.4 & \\
\hline \multicolumn{4}{|l|}{$\operatorname{BMI}(\%)^{\mathrm{a}}\left(\mathrm{kg} / \mathrm{m}^{2}\right)$} \\
\hline$<25$ & 33.6 & 52.2 & \multirow[t]{3}{*}{$<0.001$} \\
\hline 25 to $<30$ & 40.7 & 35.1 & \\
\hline$\geq 30$ & 25.7 & 12.7 & \\
\hline Mean \pm SD age at menarche (years) & $13.7 \pm 1.8$ & $13.5 \pm 2.1$ & 0.021 \\
\hline Mean \pm SD age at spontaneous menopause (years) & $50.5 \pm 3.7$ & $50.4 \pm 3.9$ & 0.711 \\
\hline Nulliparity (\%) & 5.3 & 3.4 & 0.089 \\
\hline Mean \pm SD number of full-term pregnancies ${ }^{b}$ & $1.8 \pm 0.9$ & $1.7 \pm 0.9$ & 0.127 \\
\hline Mean \pm SD age at first delivery (years) ${ }^{b}$ & $24.0 \pm 4.6$ & $24.9 \pm 4.8$ & 0.012 \\
\hline Women that breastfed (\%) & 86.4 & 90.3 & 0.344 \\
\hline Mean \pm SD duration of breastfeeding (months) ${ }^{c}$ & $8.1 \pm 8.7$ & $7.7 \pm 7.4$ & 0.269 \\
\hline OC use $(\%)$ & 42.1 & 54.7 & 0.001 \\
\hline \multicolumn{4}{|l|}{ Duration of OC use $(\%)$ (years) } \\
\hline 0 to $<1$ & 57.9 & 45.3 & \multirow[t]{4}{*}{0.002} \\
\hline 1 to $<5$ & 14.4 & 22.7 & \\
\hline 5 to $<10$ & 12.7 & 17.1 & \\
\hline$\geq 10$ & 15.0 & 14.9 & \\
\hline HRT use $(\%)$ & 29.6 & 65.8 & $<0.001$ \\
\hline \multicolumn{4}{|l|}{ Duration of HRT use (\%) (years) } \\
\hline 0 to $<1$ & 70.4 & 34.2 & \multirow[t]{3}{*}{$<0.001$} \\
\hline 1 to $<5$ & 14.3 & 33.1 & \\
\hline$\geq 5$ & 15.3 & 32.7 & \\
\hline \multicolumn{4}{|l|}{ Regimen of HRT $(\%)^{\mathrm{d}}$} \\
\hline Combined, estrogen plus progestin & 71.2 & 67.8 & \multirow[t]{2}{*}{0.487} \\
\hline Estrogen only & 28.8 & 32.2 & \\
\hline First degree family history of breast or ovarian cancer $(\%)$ & 18.1 & 15.5 & 0.138 \\
\hline Smoking $(\%)$ & 20.2 & 15.9 & 0.041 \\
\hline
\end{tabular}

${ }^{\mathrm{a}}$ Calculated as weight in $\mathrm{kg}$ divided by height in $\mathrm{m}^{2}$ at the time of the diagnosis. ${ }^{\mathrm{b}}$ Among women who had a full-term pregnancy. ${ }^{\mathrm{c}}$ Among those who ever breastfed. ${ }^{\mathrm{d}}$ Among those who ever used HRT.

interactions with breast cancer risk. The analysis revealed no indication for increased risk of breast cancer with HRT use. On the contrary, HRT use was associated with a decrease in breast cancer risk ( 1 to $<5$ years of HRT use: OR $0.22,95 \% \mathrm{CI}$ $0.14-0.32$; $\geq 5$ years of HRT use: OR $0.23,95 \%$ CI $0.16-0.34)$.

This is inconsistent with previous studies suggesting that HRT use for $>5$ years is associated with a small but significant increase in the risk of breast cancer (6-10). The extremely opposite trend in the present study might be due to preferential participation in the study by controls with these breast cancer risk factors present over those without these factors. It should be noted that although HRT use (considering the entire study population from the parent study involving control women that did not provide blood samples) was also associated with a decreased breast cancer risk $(<1$ year of HRT use vs. never users: OR $0.46,95 \%$ CI $0.26-0.83 ; 1$ to $<5$ years of HRT use vs. never users: OR $0.44,95 \%$ CI $0.26-0.73$; $\geq 5$ years of HRT use vs. never users: OR 0.51, 95\% CI 0.30-0.87), yet it proved to be slightly less. Another explanation may involve the use of retrospectively collected exposure data and, similar to most observational studies, we relied on self-reports of HRT use. Furthermore, the prevalence of HRT use is highly associated with socioeconomic status; the women of higher socioeconomic status are $>3$ times more likely to undergo HRT (11). In our study population, $10.1 \%$ of cases and $23.4 \%$ of controls reported having a university degree or $\mathrm{PhD}$, whereas for 
Table II. HRT use and risk of breast cancer.

\begin{tabular}{|c|c|c|c|c|}
\hline Duration of HRT use (years) & Cases, n (\%) & Controls, n (\%) & $\mathrm{OR}^{\mathrm{a}}(95 \% \mathrm{CI})$ & P-value \\
\hline $0<1$ & $373(70.4)$ & $92(34.2)$ & 1.0 & $<0.001$ \\
\hline $1<5$ & $76(14.3)$ & $89(33.1)$ & $0.22(0.14-0.32)$ & \\
\hline$\geq 5$ & $81(15.3)$ & $88(32.7)$ & $0.23(0.16-0.34)$ & \\
\hline
\end{tabular}

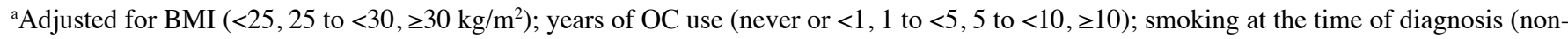
smokers, 1 to $<10$ cigarettes per day, $\geq 10$ cigarettes per day). Statistically significant results are shown in bold.

Table III. HRT use, genetic variation and risk of breast cancer.

\begin{tabular}{|c|c|c|c|c|c|c|}
\hline \multirow[b]{3}{*}{ Genotype } & \multicolumn{6}{|c|}{ HRT use (years) } \\
\hline & \multicolumn{2}{|c|}{0 to $<1$} & \multicolumn{2}{|c|}{1 to $<5$} & \multicolumn{2}{|c|}{$\geq 5$} \\
\hline & Cases/controls & $\mathrm{OR}^{\mathrm{a}}(95 \% \mathrm{CI})$ & Cases/controls & $\mathrm{OR}^{\mathrm{a}}(95 \% \mathrm{CI})$ & Cases/controls & $\mathrm{OR}^{\mathrm{a}}(95 \% \mathrm{CI})$ \\
\hline \multicolumn{7}{|l|}{ CYP1B1 } \\
\hline $\mathrm{CC}$ & $104 / 30$ & 1.0 & $25 / 36$ & $0.2(0.1-0.4)$ & $28 / 31$ & $0.2(0.1-0.5)$ \\
\hline $\mathrm{CG} / \mathrm{GG}$ & $263 / 62$ & $1.2(0.7-1.9)$ & $50 / 53$ & $0.3(0.2-0.5)$ & $53 / 57$ & $0.3(0.2-0.5)$ \\
\hline $\mathrm{P}_{\text {interaction }}$ & 0.884 & & & & & \\
\hline \multicolumn{7}{|l|}{ COMT } \\
\hline GG & $85 / 26$ & 1.0 & $16 / 23$ & $0.2(0.1-0.5)$ & $22 / 18$ & $0.4(0.2-0.9)$ \\
\hline GA/AA & $288 / 66$ & $1.4(0.8-2.3)$ & $60 / 66$ & $0.3(0.2-0.5)$ & $59 / 70$ & $0.3(0.2-0.5)$ \\
\hline $\mathrm{P}_{\text {interaction }}$ & 0.325 & & & & & \\
\hline \multicolumn{7}{|l|}{ GSTP1 } \\
\hline AA & $166 / 46$ & 1.0 & $26 / 41$ & $0.2(0.1-0.4)$ & $41 / 43$ & $0.3(0.2-0.5)$ \\
\hline $\mathrm{AG} / \mathrm{GG}$ & $207 / 46$ & $1.3(0.8-2.0)$ & $50 / 48$ & $0.3(0.2-0.5)$ & $40 / 45$ & $0.2(0.1-0.4)$ \\
\hline $\mathrm{P}_{\text {interaction }}$ & 0.399 & & & & & \\
\hline \multicolumn{7}{|l|}{ MnSOD } \\
\hline $\mathrm{TT}$ & $85 / 21$ & 1.0 & $21 / 18$ & $0.29(0.12-0.66)$ & $12 / 26$ & $0.10(0.04-0.24)$ \\
\hline $\mathrm{TC} / \mathrm{CC}$ & $288 / 71$ & $1.00(0.56-1.76)$ & $55 / 71$ & $0.20(0.11-0.37)$ & $69 / 62$ & $0.29(0.16-0.54)$ \\
\hline $\mathrm{P}_{\text {interaction }}$ & 0.034 & & & & & \\
\hline
\end{tabular}

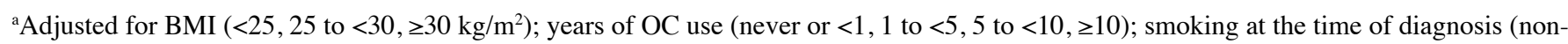
smokers, 1 to $<10$ cigarettes per day, $\geq 10$ cigarettes per day). Statistically significant results are shown in bold.

$30.7 \%$ of cases and $6.3 \%$ of controls primary school was their highest education level obtained. When analyses were adjusted for education, no significant difference in ORs was found. Nevertheless, we assume that educated women are more likely to be informed about HRT and are more aware of the breast cancer risk. Therefore, they attend breast cancer screening programs more frequently, which was also the case in our study. Additionally, before starting HRT in Slovenia, each woman should attend various screening programs (mammography, tumor marker tests) for early detection of any abnormality present and, if detected, the use of HRT is contraindicated. Thus, given the higher education level and higher prevalence of HRT use among the controls, comprehensive medical care of HRT users without preexisting breast abnormalities probably reduces the incidence of new breast cancer cases in Slovenia.
None of the 4 genetic variants studied was, by itself, statistically significantly associated with breast cancer risk. Studies to identify common genetic variants contributing to complex diseases, such as breast cancer, often yield inconsistent results $(12,13)$. Explanations include false-positive results that randomly occur, inadequate statistical power, genotype alone being an incomplete measure of the phenotypic effect and true variation in the underlying association between genotype and outcome between populations studied (14). Failure to assess exposure to environmental factors may be a further explanation for the lack of consistent findings in genetic association studies (15). This suggests that the subtle effects of some genetic variants may be magnified and only become detectable in the presence of certain exposures. Therefore, our a priori hypothesis specified that a statistically significant modification effect existed according to estrogen metabolism 
genotypes with long-term HRT use. This was confirmed by the results as a significant interaction between the MnSOD 47T >C polymorphism and HRT use was observed $\left(\mathrm{p}_{\text {interaction }}=0.036\right)$.

Manganese superoxide dismutase (MnSOD) is the major antioxidant in mitochondria involved in the first-line defense against ROS-induced oxidative damage. It catalyzes the dismutation of superoxide radicals, formed during the redox cycling between estrogen semiquinones and estrogen quinones, to form hydrogen peroxide $\left(\mathrm{H}_{2} \mathrm{O}_{2}\right)$, which is further detoxified to water by glutathione peroxidase (16). A human genetic polymorphism at codon 16 of the mitochondrial targeting sequence leads to a substitution from valine (Val) to alanine (Ala) (17). Rosenblum et al predicted that the Val to Ala substitution alters the secondary structure of the protein, which may affect the localization and transport of the enzyme into mitochondria, where it exerts its antioxidant action (18). Subsequent data supported this hypothesis, revealing that MnSOD Ala alleles were 30-40\% more efficiently localized to the mitochondrial matrix compared with MnSOD Val alleles (19). In view of these findings, it is expected that the Val form is likely to be associated with higher levels of ROS and thus predisposes to a higher risk of cancer. However, various experiments aiming to study the associations between this polymorphism and breast cancer have revealed a controversial picture. Ambrosone et al reported that the MnSOD Ala/ Ala genotype is associated with a 4.3-fold increased risk of breast cancer, particularly among premenopausal women with a low consumption of dietary sources of antioxidants (20). This is in agreement with the finding of Mitrunen et al that women with MnSOD Ala allele-containing genotypes have a 1.7-fold increased risk of breast cancer (21). Furthermore, they observed a significant interaction between HRT use and the MnSOD genotype. Women who had ever used HRT and who carried the MnSOD Ala allele-carrying genotype had a 2.5-fold increased risk of breast cancer (21).

In the present study we did not observe an increased risk of breast cancer in either short- or long-term HRT users, yet we found that the risk of breast cancer associated with long-term vs. short-term HRT use was decreased in women homozygous for the wild-type Val allele and increased among women with at least one variant allele of the MnSOD Val16Ala polymorphism. A plausible mechanism responsible for the higher risk of breast cancer associated with the Ala allele and lower risk of breast cancer associated with the Val allele has been explained by Bag and Bag (22). When MnSOD is inhibited to enter the mitochondrial matrix, as is the case with the Val form, superoxides $\left(\mathrm{O}_{2}{ }^{*}\right)$ cannot be dismutated to $\mathrm{H}_{2} \mathrm{O}_{2}$ and thus cause cellular damage, which induces the release of cytochrome $\mathrm{c}$ from the mitochondrial membrane, and consequently leads to apoptosis. Hence, although enormous cellular damage is posed by $\mathrm{O}_{2}$, which is more likely to occur by excess production of $\mathrm{O}_{2}$. formed in the case of surplus exogenous estrogens during prolonged HRT use, apoptosis may stop the occurrence of cancer. On the other hand, when MnSOD efficiently dismutates $\mathrm{O}_{2} \cdot$ to $\mathrm{H}_{2} \mathrm{O}_{2}$, the latter should be neutralized by glutathione peroxidase. If not, which again is more likely to occur upon excess production of $\mathrm{O}_{2}$ formed in the case of surplus exogenous estrogens during prolonged HRT use, $\mathrm{H}_{2} \mathrm{O}_{2}$ can react to yield other ROS, mostly hydroxyl radicals $(\cdot \mathrm{OH})$, which are highly detrimental to DNA. As increased $\mathrm{H}_{2} \mathrm{O}_{2}$ levels reduce the possibility of tumor necrosis factor- $\alpha$-mediated apoptosis, these mutations may be propagated to a new generation of cells, and this may give rise to cancer (23). Moreover, according to Ranganathan et al, MnSOD-dependent production of $\mathrm{H}_{2} \mathrm{O}_{2}$ up-regulates matrix metalloproteinase expression, which is responsible for the degradation of extracellular matrix and hence is likely to promote metastasis (24). According to this, our research finding indicating the association of the Ala form rather than the Val form of MnSOD and increased risk of breast cancer in long-term HRT users vs. short-term HRT users appears to be justified.

To date, three other studies have investigated interactions between polymorphisms in genes involved in estrogen metabolism and duration of HRT use in the development of breast cancer, but none of them evaluated interactions with the MnSOD 47T>C polymorphism. Rebbeck et al observed no statistically significant modification of the effect of HRT use on breast cancer risk by any of the polymorphisms they studied, including COMT 472G >A and CYP1B1 1294C>G (25). On the other hand, Diergaarde et al found statistically significant interactions between CYP1A1 1384A > G, CYP1A1 Msp I, CYP1B1 1294C>G, CYP1B1 1358A>G and PGR $1978 \mathrm{G}>\mathrm{T}$ polymorphisms and HRT use (26). However, inconsistent with the reported higher enzyme activity of the CYP1B1 432G variant resulting in increased formation of 4-hydroxy catechol estrogens, Diegaarde et al found that the risk increased slightly with increasing duration of HRT use among women with at least one CYP1B1 432G allele, whereas a large increase in risk was noted among women homozygous for CYP1B1 C432. Diegaarde et al also investigated interactions between COMT 472G $>$ A and GSTP1 313A $>\mathrm{G}$ polymorphisms and HRT use with no statistically significant interaction found. In the third study performed by MARIEGENICA Consortium, a total of 28 polymorphisms (including those we studied except for MnSOD 47T>C) located in 17 genes were analyzed, and the risk associated with the duration of HRT use (in years) was significantly modified by CYP1B1 142C $>\mathrm{G}$ and 355G $>\mathrm{T}$, GSTT1 del and GSTP1 341C $>$ T (27). It should be noted that Rebbeck et al defined long-term HRT use as $\geq 3$ years, Diergaarge et al $\geq 10$ years, whereas we defined it as $\geq 5$ years. Since we investigated polymorphisms involved only in estrogen metabolism, we included both combined, estrogen plus progestin, and estrogen only HRT. In contrast, Rebbeck et al and Diergaarde et al included only users of combined HRT in their analyses.

Since the present study was retrospective in design, and the data were obtained on the basis of a postal questionnaire, we cannot rule out the possibility of introducing biased study results. As reported previously, a low response rate among controls in the present study compared to the parent study resulted in the preferential participation in the present study of those postmenopausal women receiving HRT. However, it seems unlikely that the participation among HRT users differed according to the CYP1B1 rs1056836, COMT rs4680, GSTP1 rs1695 and MnSOD rs4880 genotype. Our study population was of medium size and although it is possible that some interactions would be detected in a larger sample, the narrow CIs indicate that the effect could not be substantial. The strengths of the study include homogenous study population, 
the availability of information on potential confounders and the investigation of functionally relevant genetic variants in genes, each being highly expressed in breast tissue and involved in a distinct estrogen metabolic sub-pathway. These data are consistent with biologically plausible interactions and merit further investigation of the MnSOD polymorphism in relation to HRT use and breast cancer risk.

Our results suggest that the MnSOD 47T $>$ C polymorphism in interaction with long-term HRT use may modify the risk of breast cancer. These findings can provide new important data on the combined effects of putative gene-environment interaction in the etiology of human breast cancer. Interpretation of this association must await the results of similar studies conducted in other population, and additional research is needed to evaluate the clinical relevance of this interaction. Eventually, pharmacogenomics may develop appropriate means for optimizing drug therapy with respect to patient genotype in order to ensure maximum efficacy with minimal adverse effects.

\section{Acknowledgements}

We would like to thank Ms. Mojca Pirc for revision of the English text. This study was supported by grant L3-0431 from the Slovenian Research Agency.

\section{References}

1. Yager JD and Davidson NE: Estrogen carcinogenesis in breast cancer. N Engl J Med 354: 270-282, 2006.

2. Yue W, Santen RJ, Wang JP, et al: Genotoxic metabolites of estradiol in breast: potential mechanism of estradiol-induced carcinogenesis. J Steroid Biochem Mol Biol 86: 477-486, 2003.

3. Rogan EG, Badawi AF, Devanesan PD, et al: Relative imbalances in estrogen metabolism and conjugation in breast tissue of women with carcinoma: potential biomarkers of susceptibility to cancer Carcinogenesis 24: 697-702, 2003.

4. Cavalieri E, Frenkel K, Liehr JG, Rogan E and Roy D: Estrogens as endogenous genotoxic agents - DNA adducts and mutation. J NCI Monogr 27: 75-93, 2000.

5. Pharoah PD, Tyrer J, Dunning AM, Easton DF and Ponder BA: Association between common variation in 120 candidate genes and breast cancer risk. PLoS Genet 3: e42, 2007.

6 . Breast cancer and hormone replacement therapy: collaborative reanalysis of data from 51 epidemiological studies of 52,705 women with breast cancer and 108,411 women without breast cancer: Collaborative group on hormonal factors in breast cancer. Lancet 350: 1047-1059, 1997.

7. Fournier A, Berrino F, Riboli E, Avenel V and Clavel-Chapelon F: Breast cancer risk in relation to different types of hormone replacement therapy in the E3N-EPIC cohort. Int J Cancer 114: 448-454, 2005.

8. Beral V: Million women study collaborators. Breast cancer and hormone-replacement therapy in the million women study. Lancet 362: 419-427, 2003.

9. Speroff L: The million women study and breast cancer. Maturitas 46: 1-6, 2003.

10. Anderson GL, Limacher M, Assaf AR, et al: Effects of conjugated equine estrogen in postmenopausal women with hysterectomy: the Women's Health Initiative randomized controlled trial. JAMA 291: 1701-1712, 2004
11. Finley C, Gregg EV, Solomon LJ and Gay E: Disparities in hormone replacement therapy use by socioeconomic status in a primary care population. J Community Health 26: 39-50, 2001.

12. Mitrunen K and Hirvonen A: Molecular epidemiology of sporadic breast cancer. The role of polymorphic genes involved in oestrogen biosynthesis and metabolism. Mutat Res 544: 9-41, 2003.

13. Thompson PA and Ambrosone C: Molecular epidemiology of genetic polymorphisms in estrogen metabolizing enzymes in human breast cancer. J Natl Cancer Inst Monogr 27: 125-134, 2000.

14. Colhoun HM, McKeigue PM and Davey Smith G: Problems of reporting genetic associations with complex outcomes. Lancet 361: 865-872, 2003

15. Low YL, Dunning AM, Dowsett M, et al: Implications of gene-environment interaction in studies of gene variants in breast cancer: an example of dietary isoflavones and the D356N polymorphism in the sex hormone-binding globulin gene. Cancer Res 66: 8980-8983, 2006.

16. Wang S, Wang F, Shi X, et al: Association between manganese superoxide dismutase (MnSOD) Val-9Ala polymorphism and cancer risk - A meta-analysis. Eur J Cancer 45: 2874-2881, 2009.

17. Tamimi RM, Hankinson SE, Spiegelman D, Colditz GA and Hunter DJ: Manganese superoxide dismutase polymorphism, plasma antioxidants, cigarette smoking, and risk of breast cancer. Cancer Epidemiol Biomarkers Prev 13: 989-996, 2004.

18. Rosenblum JS, Gilula N and Lerner RA: On signal sequence polymorphisms and disease of distribution. Proc Natl Acad Sci USA 93: 4471-4473, 1996.

19. Sutton A, Khoury H, Prip-Buus C, Cepanec C, Pessayre D and Degoul F: The Ala16Val genetic dimorphism modulates the import of human manganese superoxide dismutase into rat liver mitochondria. Pharmacogenetics 13: 145-157, 2003.

20. Ambrosone CB, Freudenheim JL, Thompson PA, et al: Manganese superoxide dismutase (MnSOD) genetic polymorphism, dietary antioxidants, and risk of breast cancer. Cancer Res 59: 602-606, 1999.

21. Mitrunen K, Sillanpaa P, Kataja V, et al: Association between manganese superoxide dismutase (MnSOD) gene polymorphism and breast cancer risk. Carcinogenesis 22: 827-829, 2001

22. Bag A and Bag N: Target sequence polymorphism of human manganese superoxide dismutase gene and its association with cancer risk: A review. Cancer Epidemiol Biomarkers Prev 17: 3298-3305, 2008

23. Dasgupta J, Subbaram S, Connor KM, et al: Manganese superoxide dismutase protects from TNF- $\alpha$-induced apoptosis by increasing the steady-state production of $\mathrm{H}_{2} \mathrm{O}_{2}$. Antioxid Redox Signal 8: 1295-1305, 2006.

24. Ranganathan AC, Nelson KK and Rodriguez AM: Manganese superoxide dismutase signals matrix metalloproteinase expression via $\mathrm{H}_{2} \mathrm{O}_{2}$-mediated ERK1/2 activation. J Biol Chem 276: 14264-14270, 2001.

25. Rebbeck TR, Troxel AB, Shatalova G, et al: Lack of effect modification between estrogen metabolism genotypes and combined hormone replacement therapy in postmenopausal breast cancer risk. Cancer Epidemiol Biomarkers Prev 16: 1318-1320, 2007.

26. Diergaarde B, Potter JD, Jupe ER, et al: Polymorphisms in genes involved in sex hormone metabolism, estrogen plus progestin hormone therapy use, and risk of postmenopausal breast cancer. Cancer Epidemiol Biomarkers Prev 17: 1751-1759, 2008.

27. Marie-Genica consortium on genetic susceptibility for menopausal hormone therapy-related breast cancer risk. Genetic polymorphisms in phase I and phase II enzymes and breast cancer risk associated with menopausal hormone therapy in postmenopausal women. Breast Cancer Res Treat 119: 463-467, 2010. 